\title{
Kernos
}

Revue internationale et pluridisciplinaire de religion grecque antique

$10 \mid 1997$

Varia

\section{A. VERBANCK-PIÉRARD, D. VIVIERS, Culture et Cité Louise-Marie L'Homme-Wéry}

(2) OpenEdition

Journals

Édition électronique

URL : http://journals.openedition.org/kernos/690

DOI : $10.4000 /$ kernos. 690

ISSN : 2034-7871

Éditeur

Centre international d'étude de la religion grecque antique

Édition imprimée

Date de publication : 1 janvier 1997

Pagination : 358-361

ISSN : 0776-3824

Référence électronique

Louise-Marie L'Homme-Wéry, « A. verbanck-PIÉRARd, D. viviers, Culture et Cité », Kernos [En ligne], 10 |

1997, mis en ligne le 12 avril 2011, consulté le 24 septembre 2020. URL : http://

journals.openedition.org/kernos/690; DOI : https://doi.org/10.4000/kernos.690

Kernos 
défendue jadis par $\mathrm{H}$. VAN EFFENTERRE au sujet de la libération par l'homme politique athénien du territoire d'éleusis qu'avait conquis Mégare, il l'enrichit par une série d'études approfondies qui exploitent, avec beaucoup d'ingéniosité et d'esprit critique, non seulement les témoignages des historiens anciens, mais aussi quantité de données religieuses, politiques, économiques, militaires, topographiques même, patiemment rassemblées et bien mises en perspective. En dépit de cette multiplicité d'approches, le travail est remarquablement structuré, sa méthode pouvant être qualifiée de circulaire : chacun des six premiers chapitres contribue à étayer le propos central à partir d'éclairages différents.

Si la thèse d'une reconquête par Solon sort considérablement renforcée de ces analyses, il reste à expliquer pourquoi toute l'historiographie antique a passé sous silence l'événement. Le dernier chapitre relève, de façon remarquable, ce défi en montrant comment l'histoire athénienne qui prècède et qui suit l'illustre législateur a été profondément marquée par une souillure (agos) encourue de la part de la déesse éleusinienne à la suite du sacrilège cylonien, malédiction dont on évite soigneusement de rappeler le souvenir offensant parce qu'elle passe pour avoir été la cause de plusieurs sécessions de la cité des mystères et parce qu'on continue d'en redouter les néfastes effets.

On peut prévoir que toutes les interprétations et les reconstructions habiles que propose l'A. ne rencontreront pas l'adhésion unanime des historiens spécialistes, mais l'ouvrage, très documenté et solidement argumenté, fera date. La qualité de l'information en matière religieuse et le parti nouveau que l'A. réussit à tirer des faits pour éclairer la pensée et l'œuvre soloniennes méritent en particulier l'attention. Si la bibliographie est riche, on regrettera cependant l'absence d'index.

André MOTTE

(Université de Liège)

Annie Verbanck-Pí́rard, Didier Viviers (éds), Culture et Cité. L'avènement d'Atbènes à l'époque archä̈que. Actes du Colloque international organisé à l'Université libre de Bruxelles du 25 au 27 avril 1991 par l'Institut des Hautes Études de Belgique et la Fondation Archéologique de l'U.L.B., Bruxelles, 1995. 1 vol. $17 \times 24$ cm, 252 p., 26 ill. ISBN : 2-9600094-0-1. Diffusion de Boccard.

Comme le soulignent les éditeurs, l'objet du colloque dont le présent volume publie les actes était de définir la relation entre Culture et Cité " pour mieux percevoir les conditions de l'avènement d'Athènes à l'époque archaïque ». Définissant la « culture », ils y reconnaissent " le comportement général des groupes humains, acquis par l'éducation comme par l'expérience, la paideia en grec [...] Ainsi comprise, la culture peut être bien plus qu'un révélateur de l'évolution politique et sociale d'Athènes; elle a pu également jouer un rôle actif dans cette évolution, et notamment dans la conquête et la nature de sa citoyenneté ».

Loin d'isoler Athènes en revenant à un athénocentrisme dépassé, les participants à ce colloque l'ont étudiée en confrontant son modèle à celui d'autres cités - Corinthe, Argos, les cités ioniennes -, en l'appréhendant dans la durée, de l'époque géométrique à la tyrannie qu'ils perçoivent comme une variante de structure aristocratique. Dans l'optique de déterminer les rapports entre Culture et Cité, ils ont abordé toutes les facettes de la culture athénienne, : céramique (J. BOARDMAN); pratiques littéraires avec 
la naissance de la tragédie (A. MARTIN), les épitaphes versifiées (C. SAERENS) et le rôle des rhapsodes aux Panathénées (H.A. SHAPIRO); pratiques religieuses avec l'évolution des rites funéraires (I. MORRIS), des sanctuaires (F. DE POLIGNAC), telle qu'en témoigne l'archéologie; originalité du culte d'Héraclès perceptible à travers des sources nombreuses dès le $\mathrm{VI}^{\mathrm{e}}$ siècle (A. VERBANCK-PIÉRARD); pratiques artistiques avec le fonctionnement des ateliers de potiers, peintres de vases et la détermination de leur statut (D. WILLIAMS; A.F. LAURENS), l'efflorescence de la sculpture (A.M. D'ONOFRIO) et les ateliers de sculpteurs (D. VIVIERS); et enfin origine de l'agora par J. MCK. CAMP II. E. LÉVY a clôturé ce colloque en soulignant le caractère quelque peu retardataire d'Athènes à l'époque archaïque, résultat peut-être d'un synécisme tardif.

Ce retard cependant est plus perceptible au $\mathrm{VII}^{\mathrm{e}}$ siècle où la Cité semble davantage fermée sur elle-même et où l'analyse de sa structure pose des problèmes en raison de l'absence de texte littéraire contemporain. La documentation abondante mise en œuvre par les spécialistes des différentes disciplines dans ce colloque met, au contraire, en évidence son démarrage rapide $a u v{ }^{e}$ siècle et son caractère de plus en plus ouvert aux influences étrangères. Comme le montre J. BOARDMAN, c'est Corinthe, dans la première moitié du siècle, l'Ionie, dans la seconde, qui influencent de manière déterminante les artistes athéniens, mais sans qu'il y ait à Athènes un style civique unitaire. L'étude des ateliers de sculpteurs par D. VIVIERS met, en effet, en évidence qu'il n'y a pas un "style » attique, mais une multiplicité d'approches stylistiques, qui répondent à l'étroitessse des liens entre clientèle et esthétique. L'absence d'un style homogène caractérisant la Cité dans son ensemble, tel qu'on en rencontre par exemple à Corinthe, Samos, Milet, semble, constate D. VIVIERS, traduire l'individualité du citoyen face à l'idée d'unité politique qui tente de s'implanter.

D'autres facteurs cependant - d'ordre religieux et moraux - développent un système de valeur dans lequel l'idéal aristocratique se fait au $\mathrm{vI}^{\mathrm{e}}$ siècle populaire. L'organisation de récitations par les rhapsodes de poèmes du cercle épique a lieu dès 566, dans les Panathénées, ainsi que le montre H.A. SHAPIRO par les représentations figurant sur les peintures de vases. Elle diffuse dans le dèmos un idéal jusque-là aristocratique, comme le souligne E. LÉvY. Elle soude ainsi les Athéniens, haussant le peuple au niveau de valeurs aristocratiques qui deviennent progressivement les siennes et pose, sans le vouloir ni le savoir, les bases de la future démocratie à l'intérieur même du régime tyrannique. Le culte d'Héraclès de même unifie. A. VERBANCK-PIÉRARD montre son importance à Athènes dès les premiers vases à figures noires, et, par une étude systématique de la documentation, met en évidence les traits apparemment contradictoires d'un héros-dieu qui, tout en étant ressenti comme "extérieur », est fortement enraciné dans le terroir local. Héraclès participe ainsi à l'« invention d'Athènes » et « porte en germe tout le dynamisme de la cité en formation qui ne peut, sous peine d'échec stérile, se passer d'admettre la pluralité ». A. VERBANCK-PIÉRARD en conclut que le cultuel façonne ici le politique, tout autant qu'il le révèle. L'étude d' A. MARTIN sur les premiers tragiques - Thespis, Choirilos, Phrynichos - montre par ailleurs qu'il n'y a pas, sous la tyrannie, d'inféodation du culturel au politique, si du moins on en juge par les titres et les sujets des ouvres de ces premiers tragiques qui ne témoignent pas de leur mise au service des Pisistratides.

C'est une position quelque peu différente qu'adopte I. MORRIs dans son étude des rites funéraires en Attique, à partir des données archéologiques, de l'époque géométrique au début du $\mathrm{v}^{\mathrm{e}}$ siècle. Redéveloppant d'une manière originale le point de vue 
qu'il a défendu depuis 1987, dans Burial and Ancient Society. The Rise of the Greek City-State, I. MORRIS défend l'idée d'une corrélation entre incinération et pouvoir aristocratique, inhumation et inclusion du dèmos dans une polis élargie. Selon lui, l'exclusion presque totale des enfants de funérailles visibles sur le plan archéologique, que l'on peut constater par une étude systématique des cimetières de l'Attique entre 1050 et 750 , aurait sa correspondance dans l'exclusion durant cette même période de la classe inférieure des $k a k o i$, qui comprendrait probablement plus de la moitié de la population de l'Attique. Seuls les agatboi auraient eu une tombe laissant archéologiquement des traces.

Les adultes décédés étant à cette époque incinérés dans des urnes, MORRIS définit ce rite comme aristocratique, tandis qu'il voit dans l'introduction vers 750 de l'inhumation et de l'incinération primaire selon laquelle le corps est brûlé dans la fosse ellemême, une innovation qui traduirait l'intégration des kakoi dans la polis. Cette intégration aurait sa correspondance dans les funérailles visibles désormais accordées aux enfants et adolescents qui continuent à être inhumés. Enfin le changement radical qui survient ca 700 dans le nombre des tombes qui diminue alors fortement - tant pour les adultes que pour les enfants - traduirait une recrudescence aristocratique, les corps étant alors systématiquement l'objet d'une incinération primaire. Ce n'est pas avant 550 avec un nouveau retour à l'inhumation que l'intégration sociale des kakoi dans la cité aurait commencé à être opérée.

Si les données avancées par I. MORRIS concernant l'absence de funérailles visibles pour les enfants durant les Siècles Obscurs sont convaincantes, on hésitera cependant à établir un lien aussi ferme qu'il ne le fait entre rites funéraires et société. Car la diminution du nombre des tombes tant pour les adultes que pour les enfants que l'on constate à partir de 700 environ a sa correspondance dans la diminution du nombre des habitations à la même époque (Morris 1987, fig. 54). L'interprétation de l'A. selon laquelle à cette même époque, les habitats n'auraient pas laissé de trace visible semble par ailleurs peu convaincante dans la mesure où l'on constate la même évolution à Argos, mais pas à Corinthe, cité dont MORRIS souligne le dynamisme: dès 800 , elle s'oriente vers la recherche des métaux dans l'Adriatique. Dès lors, on sera tenté de revenir à la thèse défendue préalablement par A. SNODGRASS et rejetée par I. MORRIS selon laquelle Athènes comme Argos sans doute aurait connu vers 700 une baisse très rapide de sa population. Celle-ci est peut-être la conséquence d'années de mauvaise récolte provoquant un réajustement brutal de sa population avec les possibilités d'exploitation de son territoire, à une époque où Athènes ne s'est pas encore tournée comme Corinthe vers des contacts extérieurs, ni vers la colonisation.

Le développement de cultes de sommet au vII ${ }^{\mathrm{e}}$ siècle, au Parnès et à l'Hymette où ils existaient dès le Géométrique ancien, et en de nombreux autres sites du Nord, du centre et de l'Est de l'Attique (Turkovouni, Merenda, Pani, Keratovouni, Prof. Ilias, Agrieliki) pourrait témoigner de cette même période de crise agraire entraînant une régression démographique. Dans l'étude fouillée de l'archéologie des sanctuaires de l'Attique géométrique et archaïque qu'il a proposée au Colloque, F. DE POLIGNAC souligne en effet le caractère à l'origine agraire de ces cultes paysans généralement voués à un Zeus pourvoyeur de pluie. Mais il note aussi la transformation que l'utilisation de l'écriture leur fait subir à partir de 650 : la présence de nombreux graffiti sur les vases modestes qui y sont alors offerts en abondance témoigne, semble-t-il, de la volonté que les paysans qui fréquentaient ces sanctuaires avaient de signaler leur identité par 
l'écriture, et d'afficher ainsi leur existence sur le plan social à une époque où politiquement cette existence ne leur était pas reconnue. Et ce n'est sans doute pas un hasard si les graffiti sur les offrandes de ces sanctuaires disparaissent, comme le souligne l'A., au début du $\mathrm{VI}^{\mathrm{e}}$ siècle, à l'époque même où l'« écriture » de la loi par Solon résout partiellement du moins les problèmes posés par une justice jusque-là aristocratique, assurant ainsi aux kakoi le statut des bomoioi qui était le leur dans l'armée, sans l'être dans la cité. Ici, plus peut-être que dans l'évolution des rites funéraires, on voit se dessiner une corrélation étroite entre le religieux, le social et le politique.

C'est cette même corrélation que l'on peut établir, me semble-t-il, si l'on estime que sur l'Agora d'Athènes les premières fondations qui remontent à Solon sont celles d'un Bouleutèrion-Mètrôon qui continuera à y exister jusqu'à la fin de l'antiquité, en association avec l'usage de l'écriture dans la vie politique, puisqu'à Athènes, le Mêtrôon était centre des archives civiques. Dans son étude sur les origines de l'Agora qui clôt les Actes de ce colloque, J. MCK. CAMP II développe cependant l'idée d'une fonction d'abord athlétique et sportive pour le site, la découverte en 1980 d'une piste dont le départ se trouvait à l'Est de l'autel des Douze dieux l'amenant à voir dans le vaste espace dégagé situé à l'Est du Colonos Agoraios un terrain d'entraînement à la fois sportif et militaire. Mais ici encore la présence d'un autel aux Douze Olympiens, fils d'une Mère olympienne, imprime sa marque au site et en ramène, je pense, les origines à Solon. Des inscriptions des premiers siècles de notre ère témoignent d'ailleurs des liens qui, à Athènes, unissent encore à cette époque les éphèbes à la Mère.

On regrettera dans ce colloque par ailleurs si riche l'absence de la publication de l'étude de M. STAHL sur l'Eunomie solonienne qui aurait pu montrer l'importance de la pensée politique de Solon pour façonner cet idéal politique qui, à Athènes, consista à faire partager par le dèmos des valeurs jusque-là aristocratiques. L'étude des épitaphes versifiées par C. SAERENS montre cependant que, dans l'Athènes du $\mathrm{VI}^{\mathrm{e}}$ siècle, la poésie est perçue comme source de pérennité.

Louise-Marie L'Homme-Wéry

(Université de Liège)

\section{Marcel Piérart, Gilles Touchais, Argos. Une ville grecque de 6000 ans,} Paris-Méditerranée, CNRS Éditions, 1996, 127 p., nbr. ill., plans, cartes (Patrimoine de la Méditerranée). ISBN : 2-271-054222-2.

Célèbre et mal connue, telle se présente Argos, en une formulation paradoxale qui, à elle seule, suffirait à justifier l'ample parcours des auteurs à travers six millénaires d'histoire. Le déséquilibre entre l'étendue de la période prise en compte et les modestes dimensions du volume pourrait paraître problématique : ces craintes sont injustifiées lorsqu'on en mesure la clarté, le caractère didactique et le souci constant d'offrir le dernier état des connaissances sur Argos, en faisant la part de l'hypothèse, sans pour autant verser dans des polémiques de spécialistes. En douze chapitres bien équilibrés, c'est toute l'histoire d'Argos qui déroule sa trame, depuis le temps des héros jusqu'à nos jours. Illustrations et plans ne font pas qu'agrémenter la lecture : ils la nourrissent par la présentation d'objets inédits et par des reconstitutions en élévation. La perspective adoptée est résolument événementielle, ce qui donne aux époques classique et hellénistique de cette histoire de la cité la dimension d'une fresque guerrière. Au cour d'enjeux qu'elle maîtrise ou qui la dépassent, Argos est bien cette «ville grecque 